\title{
Euclidean Nets: An Automatic and Reversible Geometric Smoothing of Discrete 3D Object Boundaries
}

\author{
Achille J.-P. Braquelaire and Arnault Pousset \\ LaBRI - Laboratoire Bordelais de Recherche en Informatique - UMR 5800, Université \\ Bordeaux 1 \\ 351 cours de la Libération, F-33405 Talence, France \\ \{Braquelaire, Arnault.Pousset\}@labri.u-bordeaux.fr
}

\begin{abstract}
In this work we describe a geometric method to smooth the boundary of a discrete $3 \mathrm{D}$ object. The method is reversible in the sense that the discrete boundary can be retrieved by digitizing the smoothed one. To this end, we propose a representation of the boundary of a discrete volume that we call Euclidean net and which is a generalization to the three-dimensional space of Euclidean Path introduced by Braquelaire and Vialard [4]. Euclidean nets can be associated either to voxel based boundaries or to inter-voxel based boundaries. In this paper we focus on the first approach.
\end{abstract}

Keywords: Digital Surfaces. Discrete boundary representation. Smoothing.

\section{Introduction}

Processing three-dimensional discrete images such as X-ray computer tomography images or MRI brings up the problem of defining and representing threedimensional discrete objects [11]. Usually a three-dimensional discrete image (in short 3D image) is a parallelepipedic matrix of voxels and a three-dimensional discrete object (in short 3D object) of a 3D image is defined as a set of connected component of voxels of this image. In this work, we call discrete object, or simply object, any 6-connected set of voxels. For instance a raw visualization of a discrete sphere (a maximal object which voxel centers are inside a Euclidean sphere), also called cuberille representation [10], can be seen in Fig 1 (a).

There are several motivations to build an effective representation of the boundary of a $3 \mathrm{D}$ object. One of them is that a boundary representation fits with conventional rendering techniques. Another one is that the encoding of a 3D object with a matrix of labels does not provide a convenient framework for image analysis applications such as the extraction of geometric and topological feature or object recognition.

A first approach, known as the marching cubes algorithm [13], provides a method for extracting a polygonal surface mesh from the object. Each polygon

G. Borgefors, I. Nyström, and G. Sanniti di Baja (Eds.): DGCI 2000, LNCS 1953, pp. 198-209, 2000. (C) Springer-Verlag Berlin Heidelberg 2000 
is defined according to a local configurations of height adjacent centers of voxels. This method is easy to implement but has several drawbacks both from the geometrical and the topological points of view. A second approach known as active surfaces consists in moving the control points of a parametric surface enclosing the digital object in order to minimize an energy function [20,21]. With both these approaches the boundary of the discrete object is described by a continuous surface defined in the Euclidean space $\mathbb{R}^{3}$.

An alternative method is to use the digital surface (or discrete surface) of the object. Two approaches are commonly used : the voxel based approach $[15,7,14,22]$ and the inter-voxel based approach $[17,8,4]$. It has been shown that the inter-voxel surface can be seen as a marching-cube algorithm define with an alternative interpolation scheme [12].

Several methods have been proposed to associate a surface mesh with the discrete surface of an object. For instance the set of centers of surface voxels can be triangulated by linking together some adjacent centers [24,22]. In Fig.1 (b) is displayed a wire-frame representation of the surface mesh computed by the method proposed by Thuermer [22], and in Fig.1 (c) is displayed a surface rendering of this surface mesh. The aspect of the rendered surface mesh is smoother than the cuberille representation but is still far from the aspect of a continuous sphere. A usual approach to improve the visualization of a digital surface is to estimate a normal at each point of the discrete surface and to perform a shading (for instance a usual Gouraud shading [9]).

Here we propose an alternative approach based on a geometrical smoothing of the digital surface. In general a discrete 3D image comes from the digitizing of a continuous $3 \mathrm{D}$ scene. In this context a discrete object is also the result of the digitizing of a continuous one. It is thus natural to try to retrieve from the boundary of a discrete object an approximation of the boundary of the underlying continuous object. The goal of the geometric smoothing is to approximate this underlying continuous boundary by performing local movings of the surface points. An example of geometric smoothing of a discrete sphere is shown in Fig.1 (d). Each point of the triangulated discrete surface has been moved according to a method described in the following of the paper.

This method is a generalization to the three-dimensional case of the method of Euclidean paths introduced by Braquelaire and Vialard [27,4] to smooth the discrete boundary of a discrete $2 \mathrm{D}$ region. A Euclidean path is associated to a discrete 4-connected or 8-connected path by moving each point of the discrete path from its position to any position of the open unit square centered on it. A method has been proposed to compute the moving of each point of the discrete path laying on the computation of a discrete tangent at each of these points. It has been shown that so called tangent driven Euclidean paths highly improve the visual aspect of a discrete region boundary and provides a convenient representation for geometric transformations and a good estimation of geometric features (tangent and normal, perimeter) [25]. The smoothing preserve the topology of the set of boundaries of a segmented image [1]. This method has been successfully used to solve some image processing problems [3,26]. 


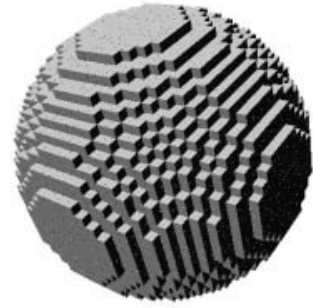

(a)

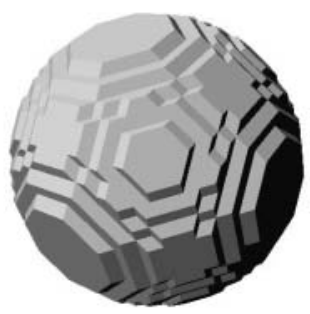

(c)

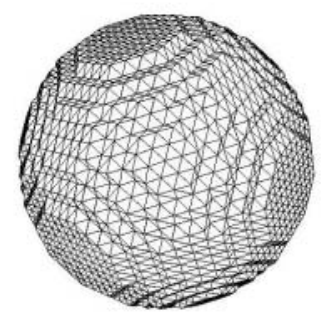

(b)

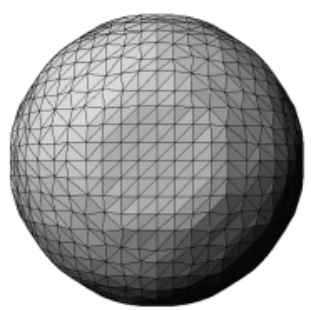

(d)

Fig. 1. Four representations of a discrete sphere: (a) cuberille representation, (b) wire-frame representation of a surface mesh associated with the discrete surface, (c) surface rendering of the surface mesh, (d) geometric smoothing of the discrete surface

The aim of this work is to propose a similar method to smooth the discrete surface of a 3D object. A geometrical analysis is performed at each point of the discrete surface providing an estimation of a tangent plane. The point is then projected onto this plane. This method is reversible in the sense that the discrete surface can be retrieved by digitizing the smoothed one. Moreover, this method computes a canonical result and does not need any manual parameterization.

In the following section we introduce discrete and Euclidean nets which are the 3D analogous of 2D discrete and Euclidean paths. In Section 3 we describe the model of tangent driven Euclidean nets. In Section 4 we address the problem of computing both a tangent plane an a normal vector to a point of a discrete surface. Finally in Section 5 we present both qualitative and quantitative results of experiments of geometric smoothing.

\section{Discrete and Euclidean Nets}

Let us now give some basic definitions used in the following. The coordinates of a point $P$ are denoted by the tuple $\left(x_{P}, y_{P}, z_{P}\right)$. The set $\mathbb{Z}^{3}$ of points with integer coordinates is called the discrete space and its elements are called discrete 
points. We denote by upper case letters the points of the discrete space $\mathbb{Z}^{3}$ and in lower case letters the points of the Euclidean space $\mathbb{R}^{3}$.

A voxel is a colored unit cube the center of which is a discrete point. The coordinates of a voxel are the coordinates of its center. An image is a set of voxels and the image domain is the set of voxel centers. In the following we consider images the domain of which is a parallelepipedic set of discrete points. The voxel based approach has been chosen to define the boundary of an object. More precisely, the discrete surface of an object $V$ is the subset $S(V)$ of $V$ such that each voxel of $S(V)$ is 6-connected to at least a voxel of the complement of $V$ in the image. A voxel of $S(V)$ is called a surface voxel of $V$.

The following definitions are extensions for the three dimensional case of the definitions used in the model of Euclidean paths [4].

Definition 1. Let $P=\left(x_{P}, y_{P}, z_{P}\right)$ be a discrete point. The cell of $P$ is the set of points $p$ of $\mathbb{R}^{3}$ verifying: $\left|x_{P}-x_{p}\right|<\frac{1}{2},\left|y_{P}-y_{p}\right|<\frac{1}{2},\left|z_{P}-z_{p}\right|<\frac{1}{2}$.

The cell of a discrete point $P$ is thus the open unite cube centered on $P$.

Definition 2. Let $P$ be a discrete point. We say that $p$ is a Euclidean point associated with $P$ if $p$ belongs to the cell of $P$.

In two dimensions it is natural to define the boundary of a discrete region by a discrete path (the nodes of the path being either pixel or inter-pixel positions). In three dimensions the analogous of a discrete path is a graph of discrete point which edges link neighboring elements of the boundary according to the considered topology. This graph describes the polygonal decomposition of the boundary. Since we consider here voxel based boundaries the edges of this graph link centers of adjacent boundary voxels. We do not focus in the following on the definition and the construction of this graph (any topological consistent definition can be used). Our experiments have been done by using the decomposition described by Thuermer [22].

Definition 3. A discrete net is a graph associated which a connected component of the polygonal decomposition of the boundary of a $3 D$ object. A Euclidean net associated with a discrete net $N$ is the graph obtained by replacing in $N$ each discrete point by an associated Euclidean point.

The transform from a discrete point to an associated Euclidean one can be reversed simply by rounding the coordinates of the Euclidean point. The transform from discrete nets to Euclidean nets is thus also reversible. Since there is an infinity of Euclidean nets that can be associated with a discrete one, we must now define a strategy to associate a discrete point with to a Euclidean one. Recall that the goal of the geometric smoothing is to approximate the underlying continuous boundary. Good results were obtained in the 2D case by projecting the discrete point on the support of an arithmetic line tangent to the boundary. In the following section we describe a analogous 3D method based on arithmetic digital planes introduced by Reveilles [16]. 


\section{Tangent Plane Driven Euclidean Nets}

Let us first recall the following definition:

Definition 4. [16] $A$ discrete naive plane (in short discrete plane) is a set of points $(x, y, z)$ of $\mathbb{Z}^{3}$ satisfying the double inequality $\mu \leq a x+b y+c z<\mu+\omega$, with $a, b, c, \mu, \omega \in \mathbb{Z}$ and $\omega=\max (|a|,|b|,|c|)$.

The coefficients $(a, b, c, \mu)$ are called the characteristics of the the discrete plane. The vector of coordinates $(a, b, c)$ is the normal vector of the discrete plane. The coefficient $\mu$ describes the position of the discrete plane.

Definition 5. Let $\mathcal{P}$ be a discrete plane of characteristic $(a, b, c, \mu)$. The lower and upper leaning planes of $\mathcal{P}$ are the real planes defined respectively by the equations $a x+b y+c z=\mu$ and $a x+b y+c z=\mu+\omega-1$.

Definition 6. Let $\mathcal{P}$ be a discrete plane of characteristic $(a, b, c, \mu)$. The centered plane of $\mathcal{P}$ is the real plane $\overline{\mathcal{P}}$ defined by the equation $a x+b y+c z=\mu+\frac{\omega-1}{2}$.

The upper and lower leaning planes of a discrete plane $\mathcal{P}$ bound the set of Euclidean points which can be associated with $\mathcal{P}$. Thus the centered plane is an average plane among all the real planes associated with $\mathcal{P}$. We suppose for the rest of this paper that $0 \leq a \leq b \leq c$ and $0<c$. Thus we have $\omega=c$. The normal vector of such a discrete plane belongs to a $48^{\text {th }}$ of space called standard simplex [6]. The other cases can be obtained by symmetry.

The strategy used to associate a Euclidean point $p$ with a point $P$ of a discrete net can be summarized as follows :

1. Select a discrete plane $\mathcal{P}(P)$ containing $P$.

2. Project $P$ onto the centered plane of $\mathcal{P}(P)$ such that the projected point $p$ satisfies the conditions of Definition 1.

There are several ways to select the plane $\mathcal{P}(P)$. It seams natural to try to find the discrete plane which is the better approximation of the Euclidean plane tangent to the underlying real boundary. We address the problem of the selection of a discrete tangent plane in the following section. For now we call tangent plane at $P$ any discrete plane that contains $P$.

Definition 7. Let $P$ be a discrete point and let $\mathcal{P}$ be a discrete plane of characteristics $(a, b, c, \mu)$ and containing $P$. Let $\overline{\mathcal{P}}$ the centered plane of $\mathcal{P}$. The canonical projection of $P$ according to $\mathcal{P}$ is the point $p$ of $\overline{\mathcal{P}}$ satisfying $\overrightarrow{P p}=\alpha\left(\begin{array}{l}a \\ b \\ c\end{array}\right)$.

From Definition 7 we have $x_{p}=\alpha a, y_{p}=\alpha b$, and $z_{p}=\alpha c$. Since $p \in \overline{\mathcal{P}}$ and by considering that $P$ is the origin of the coordinate system we have $a x_{p}+b y_{p}+c z_{p}=$ $\mu+\frac{c-1}{2}$, and thus $\alpha a^{2}+\alpha b^{2}+\alpha c^{2}=\mu+\frac{c-1}{2}$. Since we have supposed that $c>0$, we finally get:

$$
\alpha=\frac{\mu+\frac{c-1}{2}}{a^{2}+b^{2}+c^{2}}
$$


Theorem 1. According to the previous definitions the canonical projection of a point $P$ is a Euclidean point associated with $P$.

Proof. See appendix A

\section{Selection of the Tangent Plane}

The previous result states that the canonical projection of $P$ on the centered plane of a discrete plane containing $P$ is a Euclidean point associated with $P$. We have now to define a strategy to select a plane $\mathcal{P}(P)$ at each point of the discrete net. Several method have been proposed to estimate a tangent plane at a point of a discrete surface. In this work we have retained an approach based on the analysis of local configurations called tricubes. This choice is motivated by the fact that tricubes provide a simple and robust way to estimate a discrete tangent plane. Of course it would have been interesting to consider more general configurations like for instance $(n, m)$-cubes [29]. But in this work we have focused on the problem of providing a framework for reversible geometric smoothing in which any tangent plane method can be used. Improvement of methods of determination of tangent plane is out of the scope of this work.

Tricubes were introduced by Debled [6] and are the topic of several recent works $[5,18,29,28]$. A point $P$ of a digital plane $\mathcal{P}$ has exactly height neighbors. The tricube of $P$ is then the set of $P$ and of its height neighbors. The point $P$ is the center of the tricube. In the standard simplex there are exactly 40 configurations of tricubes.

For each point $P$ of the discrete net we search for local configurations of tricubes centered at $P$ and included in the net. When considering not only the standard simplex but the whole discrete space, some points of the discrete net can be associate with more than one center of tricube. There is an example of such a case in Fig. 2. When such a case arises we compute separately a Euclidean point for each tricube and we retain as Euclidean point the average of these points. Conversely there are configurations where some discrete points cannot be associated with any tricubes. For instance, there are only $58 \%$ surface voxels of a discrete sphere of radius 3 that can be associated with a tricube. This amount is of $84 \%$ for a sphere of radius 9 , and is close to $100 \%$ for spheres which radius greater than 10 voxels. When such a case arises, the point may be either left unmoved or treated during a second step. If the second option is retained the associated Euclidean point is computed by interpolating Euclidean points associated with neighbors of the unmoved point.

The example shown in Fig.1(d) has been computed with this method. Of course, the quality of the smoothing highly depends on the quality of the method used to compute the tangent plane. The method used in this work is based on the analysis of small neighborhoods. The geometrical smoothing can be improved by improving the tangent plane recognition method. 


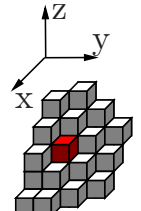

(a)

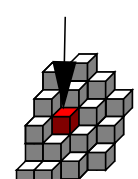

(b)

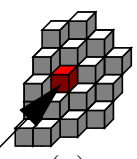

(c)

Fig. 2. The dark voxel of the discrete surface (a) can be associated with two centers of tricube (b) and (c)

\section{Experiments}

In this section we give some quantitative and qualitative results of experiments performed with the method of tangent plane estimation described in the previous section.

Let $\sigma$ be a Euclidean sphere of radius $r$ and $S$ the associated digitized sphere (the set of voxels which the center is located inside the Euclidean sphere). For each point $P$ of $S$ we denote by $\rho(P)$ the distance from $P$ to the center of the sphere (we suppose that the center $C$ of the sphere $\sigma$ is a discrete point). We define the maximal geometrical error (resp. average geometrical error) by the maximum (resp. average) of $\rho(P)$ for all the discrete points $P$ of the boundary of $S$. In Fig. 3 (a) are displayed maximal and average errors for discrete spheres from radius 2 to radius 45 before and after smoothing. Discrete points that do not fit with a tricube are smoothed by interpolation. Both maximal and average errors are reduced by half.

In Figure 3 (b) is given the result of experiments of normal estimation. The normals have been estimated according to both the discrete net and the Euclidean associated net. The normal at a point of the net is computed by averaging the normals vectors of the adjacent facets. For each point $p$ of either the discrete net or the Euclidean associated net the error on the normal is the angle between the vector $\overrightarrow{C p}$ and the estimated normal vector. Both maximal and average errors are given for spheres from radius 2 to radius 45 . The average error with the Euclidean net is lower than three degrees. The maximal error is about ten degrees for small radius and tends toward fifteen degrees when the radius increases.

Naturally these results depend on the method used to determine the tangent plane and can be improved by using a more global method than tricubes. For instance an idea to improve the estimation of the tangent plane would be to consider stochastic methods using disconnected configuration of $(n, m)$-cubes ((n,m)-cubes [29] are configurations that generalize tricubes).

Some examples of geometrical smoothing can be seen in Fig.4. For each example the image of the first column is a flat shading of the facets of the discrete net and the image of the second column is a flat shading of the facets of 
Distance in pixels

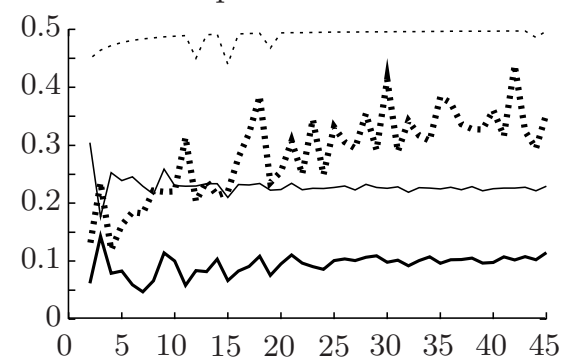

(a)

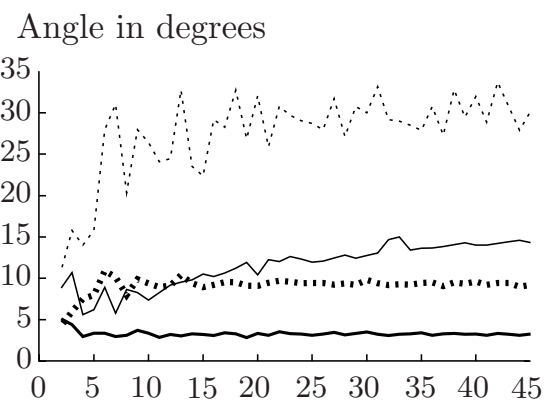

(b)

Fig. 3. (a) Maximal (dotted lines) and average (plain lines) geometrical error computed on discrete (thin lines) and smoothed (thick lines) spheres. (b) Maximal and average error of the estimated normal vectors of discrete and smoothed spheres

the associated Euclidean net. The three first rows show scenes made of regular objects (respectively a cube slightly rotated around one of its axis, a sphere of radius 20 and a combination of a plane with a sphere. The examples of the two last rows are computed from digitized 3D objects provided by the Laboratoire des Sciences de l'Image, d'Informatique et de Télédétection of Strasbourg University.

There are still visible staircase on the smoothed images that could be attenuated by improving the selection of the tangent plane. Nevertheless it is important to emphasize that the images of the second column show the result of geometric smoothing without rendering improvement. The displayed surface is exactly the smoothed surface and can be explicitly used for extract geometric features. Rendering methods based on a normal estimation [23,19] can give better visual results. On the other hand they does not provide an explicit geometric smoothing of the boundary.

\section{Conclusion}

In this work we have described a new approach to perform a discrete geometric smoothing of the boundary of a 3D object. This method extends to the threedimension case the method of Euclidean paths. Each center of voxel $P$ of the boundary is associated with a Euclidean point $p$ located inside the open unit cube centered on $P$. Given a naive plane containing $P$, the Euclidean point $p$ is the canonical projection of $P$ on the centered plane of the naive plane. By this way a Euclidean net of Euclidean points is associated with the discrete boundary.

Any definition of tangent plane can be used to computed canonical projections. Thus the accuracy of the Euclidean net construction (and of the smoothing) highly depends on the accuracy of the method used to compute the tangent plane. In this work the tangent plane at a boundary point is estimated by an- 

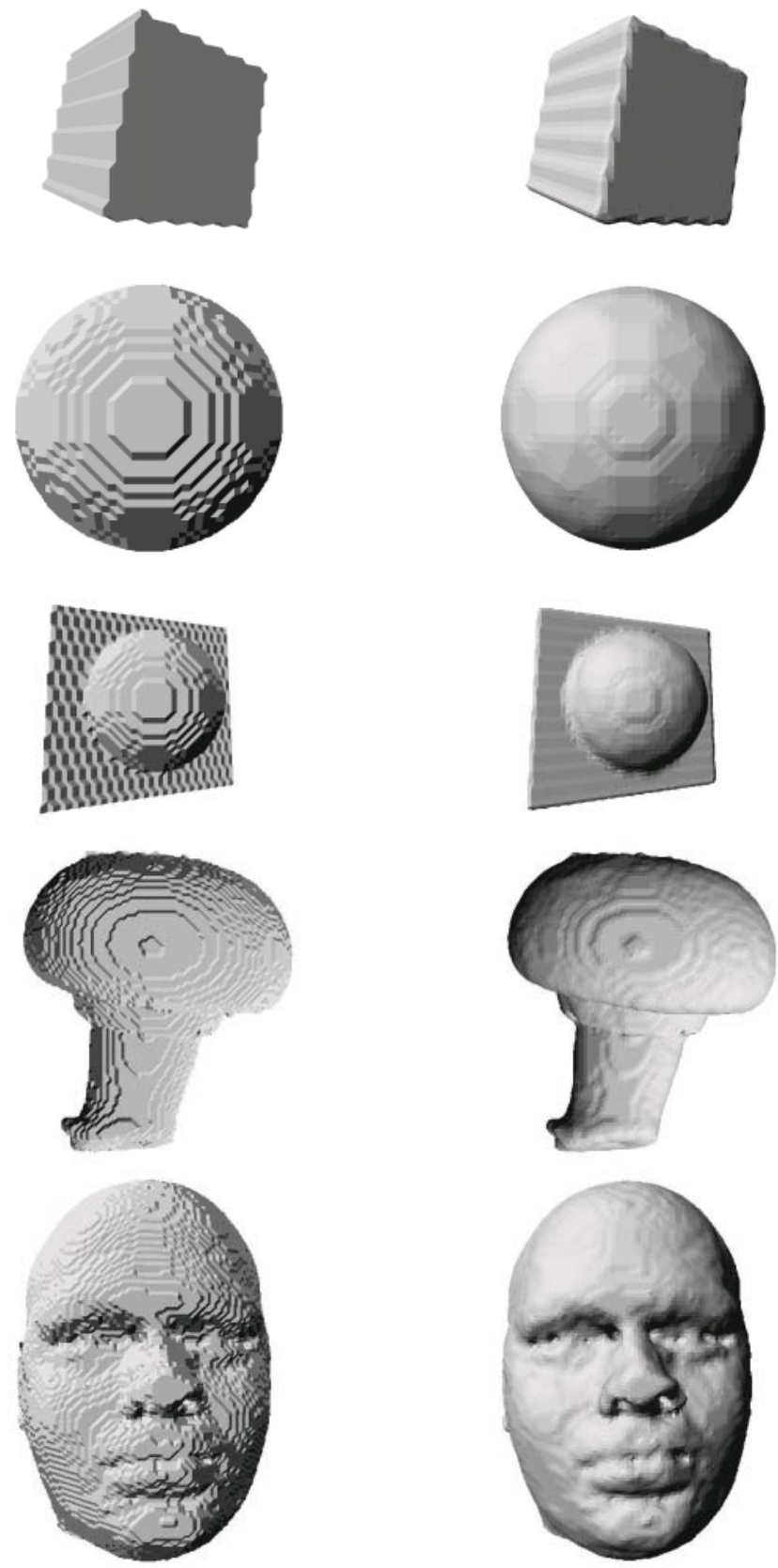

Fig. 4. Flat shading of the discrete net (first column) and of the associated Euclidean net (second column) of 3D discrete objects : a cube (first row), a sphere (second row), a combination of a plane and a sphere (third row), and digitized real objects provided by the LSIIT (fourth and fifth rows) 
alyzing tricubes configurations centered on this point. Thought this method is limited to a local analysis of the boundary around the point it gives promising results. The geometric smoothing must now be experimented with other methods of determination of tangent plane. Both quantitative and qualitative results could then be improved.

We are currently working on Euclidean nets of inter-voxel boundaries. An advantage of inter-voxel boundaries is that they provide a simple and consistent representation of the topology of the object they bounds (the discrete net is trivially obtained from the data structure). On the other hand from a geometrical point of view the inter-voxel boundaries provide a quite poor representation of the shape of the object. In that case the relevance of the Euclidean net approach is thus increased.

The method has also to be adapted to the case of a non-manifold boundary resulting from the segmentation of an aggregate of $3 \mathrm{D}$ discrete objects [2]. Finally we think that Euclidean nets will provide and efficient framework to extract geometrical parameters from a 3D object as the method of Euclidean path does for $2 \mathrm{D}$ regions [25].

\section{References}

1. J. P. Braquelaire, L. Brun, and A. Vialard. Inter-pixel euclidean paths for image analysis. In Discrete Geometry for Computer Imagery, Lecture Notes in Computer Science 1176, pages 193-204. Springer Verlag, November 1996. 199

2. J. P. Braquelaire, P. Desbarats, J. P. Domenger, and C. A. Wüthrich. A topological structuring for aggregates of 3D discrete objects. In Proc. of the 2nd IAPR-TC-15 Workshop on Graph-based Representation, pages 193-202. Österreichische Computer Gesellschaft, 1999. ISBN 3-85804-126-2. 207

3. J. P. Braquelaire and A. Vialard. A new antialiasing approach for image compositing. The Visual Computer, 13(5):218-227, 1997. 199

4. J. P. Braquelaire and A. Vialard. Euclidean paths : a new representation of boundary of discrete regions. Graphical Models and Images Processing, 61:16-43, 1999. 198, 199, 201

5. J. M. Chassery and J. Vittone. Coexistence of tricubes in digital naive plane. In Lecture Notes in Computer Science, volume 1347, pages 99-110, December 1997. 203

6. Isabelle Debled. Etude et reconnaissance des droites et plans discrets. Phd thesis, Université Louis Pasteur, Strasbourg, France, 1995. 202, 203

7. T. J. Fan, G. Medioni, and R. Nevata. Recognising 3D objects using surface descriptions. IEEE Transactions on Pattern Analisys and Machine Intelligence, 1111:1140-1157, 1989. 199

8. J. Françon. Discrete combinatorial surfaces. Grapical Models and Image Processing, $57(1): 20-26,1995.199$

9. Henri Gouraud. Continuous shading of curved surfaces. IEEE Transactions on Computers, C-20(6):623-629, June 1971. 199

10. G. T. Herman and H. K. Liu. Three-dimensionnal display of uman organs from computed tomograms. Computer Graphics and Image Processing 9, 1:1-21, 1979. 198 
11. K. H. Hohne, M. Bomans, A. Pommert, M. Riemer, C. Schiers, U. Tiede, and G. Wiebecke. 3D visualization of tomographic volume data using the generalized voxel model. The Visual Computer, 6(1):28-36, 1990. 198

12. J.-O. Lachaud and A. Montanvert. Deformable meshes with automated topology changes for coarse-to-fine 3D surface extraction. Medical Image Analysis, 3(2):187207, 1999. 199

13. W. E. Lorenson and H. E. Cline. Marching cubes: A high resolution 3D surface construction algorithm. Computer Graphics (SIGGRAPH'87), 21(4):111-118, 1987. 198

14. R. Malgouyres. A definition of surfaces of $\mathbb{Z}^{3}$ : A new discrete jordan theorem. Theoretical Computer Sciences, 186:1-41, 1997. 199

15. FD. G. Morgenthaler and A. Rosenfeld. Surfaces in three-dimensional digital images. Information and Control, 51:227-247, 1981. 199

16. J. P. Reveilles. Géométrie discrète, Calcul en nombres entiers et algorithmique. PhD thesis, Université de Strasbourg, 1991. 201, 202

17. A. Rosenfeld, T.Yung Kong, and A. Y. Wu. Digital surfaces. Grapical Models and Image Processing, 53(4):305-312, 1991. 199

18. J. M. Schramm. Coplanar tricubes. In Springer-Verlag, editor, Lecture Notes in Computer Science, volume 1347, pages 87-98, 1997. 203

19. P. Tellier and I. Debled-Rennesson. 3d discrete normal vectors. In Springer-Verlag, editor, Lecture Notes in Computer Science, volume 1568, pages 447-458, 1999. 205

20. D. Terzopoulos, A. Witkin, and M. Kass. Symmetry-seeking models and 3D object reconstruction. International Journal of Computer Vision, 1(3):211-221, 1987. 199

21. D. Terzopoulos, A. Witkin, and M. Kass. Constraints on deformable models: Recovering 3D shape and nonrigid motion. Artificial Intelligence, 36(1):91-123, 1988. 199

22. G. Thürmer. Surfaces in Three-Dimensional Discrete Space. PhD thesis, Fakultät Medien der Bauhaus-Universität Weimar, 1998. Shaker Verlag. 199, 201

23. G. Thürmer and C. A. Wüthrich. Normal computation for discrete surfaces in 3D space. Computer Graphics Forum, 16(3):15-26, 1997. Proceedings of Eurographics'97. 205

24. G. Thürmer and C. A. Wüthrich. Polygon mesh generation for discrete surfaces in 3d space. In W. Lefer and M. Grave, editors, Proc. of the Eighth Eurographics Workshop on Visualization in Scientific Computing, pages 117-126, Laboratoire d'Informatique du Littoral, Boulogne sur Mer (France), 1997. 199

25. A. Vialard. Geometrical parameters extraction from discrete paths. In Discrete Geometry for Computer Imagery, Lecture Notes in Computer Science 1176, pages 24-35. Springer Verlag, November 1996. 199, 207

26. A. Vialard. Euclidean paths for representing and transforming scanned characters. In Proceedings of GREC'97. Second IAPR Workshop on Graphics Recognition, 1997. To be published in Lecture Notes in Computer Science, Springer Verlag. 199

27. Anne Vialard. Chemins euclidiens : Un modèle de représentation des contours discrets. Phd thesis, Université Bordeaux 1, 1996. 199

28. J. Vittone. Caractérisation et reconnaissance de droites et de plans en géométrie discrète. Phd thesis, Université de Grenoble, 1999. 203

29. J. Vittone and J. M. Chassery. (n,m)-cubes and farey nets for digital naive planes understanding. In Lecture Notes in Computer Science, volume 1568, pages 76-87, 1999. 203, 204 


\section{A Proof}

We have to verify that the canonical projection $p$ satisfies the condition of Definition 2. We can suppose that the origin of the coordinates system is the discrete point $P$. Since $a \leq b \leq c$, in order to show that the point

$$
p=\left(a \frac{\mu+\frac{c-1}{2}}{a^{2}+b^{2}+c^{2}}, b \frac{\mu+\frac{c-1}{2}}{a^{2}+b^{2}+c^{2}}, c \frac{\mu+\frac{c-1}{2}}{a^{2}+b^{2}+c^{2}}\right)
$$

belongs to the unit cell centered at $P$ it suffices to verify that:

$$
\left|c \frac{\mu+\frac{c-1}{2}}{a^{2}+b^{2}+c^{2}}\right|<\frac{1}{2}
$$

The discrete tangent plane $\mathcal{P}$ is the set of points satisfying $\mu \leq a x+b y+c z \leq$ $\mu+c-1$. From the hypothesis the point of coordinates $(0,0,0)$ belongs to $P$. Thus we have from the definition of discrete plane $\mu \leq 0$ and thus

$$
\begin{aligned}
\mu & \leq 0, \\
2 \mu & <1, \\
2 \mu+c-1 & <c, \\
c(2 \mu+c-1) & <c^{2} \quad \text { since } c>0, \\
c \frac{\mu+\frac{c-1}{2}}{a^{2}+b^{2}+c^{2}} & <\frac{c^{2}}{2\left(a^{2}+b^{2}+c^{2}\right)} \quad \text { since } a^{2}+b^{2}+c^{2}>0 .
\end{aligned}
$$

Finally, since $a^{2}+b^{2}+c^{2} \geq c^{2}$ we have $c \frac{\mu+\frac{c-1}{2}}{a^{2}+b^{2}+c^{2}}<\frac{c^{2}}{2 c^{2}}$ and thus we get:

$$
c \frac{\mu+\frac{c-1}{2}}{a^{2}+b^{2}+c^{2}}<\frac{1}{2} \text {. }
$$

By a symetrical argument from $0 \leq \mu+c-1$ we get:

$$
c \frac{\mu+\frac{c-1}{2}}{a^{2}+b^{2}+c^{2}}>-\frac{1}{2} \text {. }
$$

